\title{
Preliminary trial of adjuvant surgery for advanced gastric cancer
}

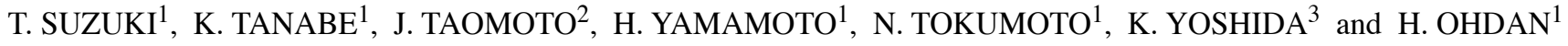 \\ ${ }^{1}$ Department of Surgery, Division of Frontier Medical Science, \\ Graduate School of Biomedical Sciences Hiroshima University, Hiroshima; ${ }^{2}$ Hiroshima City Asa Hospital, Hiroshima; \\ ${ }^{3}$ Gifu University, Graduate School of Medicine, Department of Surgical Oncology, Gifu, Japan
}

Received March 8, 2010; Accepted May 12, 2010

DOI: 10.3892/ol_00000130

\begin{abstract}
In patients with stage IV gastric cancer, systemic chemotherapy is the key treatment. Combination chemotherapy (cis-diamminedichloride platinum plus S-1 and docetaxel plus S-1) results in long-term survival in clinical practice. In selected cases, additional (adjuvant) surgery may result in further longterm survival. This study aimed to evaluate the efficacy of adjuvant surgery following the response to chemotherapy for advanced gastric cancer. Based on response to chemotherapy, the indications for adjuvant surgery (surgery after the response to chemotherapy) are that resection is expected to be curative rather than palliative, provided that no other distant metastases occur. The study included 20 advanced gastric cancer patients who had undergone gastrectomies after the response to the combination chemotherapy of docetaxel and S-1, between September 2003 and December 2008 at Hiroshima University Hospital. At a median follow-up of 980 days, the median overall survival was 855 days. A 2- and 3-year survival was observed in 80 and $54.9 \%$ of patients, respectively, following macroscopic curative surgery. In the palliative group, the median overall survival was 510 days, but a 3 -year survival was not observed. In the partial response group, the median overall survival was 865 days and a 3-year survival was observed in $37 \%$ of patients. One-year survival was not observed in the stable disease group. The patient survival in the partial response group was statistically more prolonged than in the stable disease group. The median overall survival in patients with liver metastasis was 865 days, while that in patients with peritoneal dissemination was 510 days. In conclusion, adjuvant surgery may be effective in gastric cancer patients diagnosed as stage IV by means of liver or distant lymph node metastasis, except in cases of peritoneal dissemination.
\end{abstract}

Correspondence to: Dr Kazuaki Tanabe, Department of Surgery, Division of Frontier Medical Science, Graduate School of Biomedical Sciences, Hiroshima University, 1-2-3, Kasumi, Minami-ku, Hiroshima 734-8551, Japan

E-mail: ktanabe2@hiroshima-u.ac.jp

Key words: gastric cancer, adjuvant surgery, S-1, docetaxel

\section{Introduction}

Gastric cancer is more prevalent in East Asia, Eastern Europe and Central and South America than in other countries. Worldwide, gastric cancer ranks second among all causes of death from cancer, with about 7,000,000 confirmed deaths annually (1). In Japan, it is one of the most frequent causes of cancer mortality, despite notable advances in diagnosis and treatment (2). Outcomes are extremely poor in patients with unresectable gastric cancer, with the median survival ranging from 3-5 months even with the best supportive care $(3,4)$.

Numerous randomized controlled trials of various treatment regimens have previously been reported, including 5-fluorouracil, doxorubicin and mitomycin (5), epirubicin and cisplatin (CDDP) in combination with continuous infusion of 5-fluorouracil (6) and 5-fluorouracil and cisplatin (7). However, the trials produced median survival rates of less than 1 year. Recently, two randomized controlled trials were reported from Japan $(8,9)$. One was the JCOG9912 trial, which showed S-1 to be non-inferior to continuous infusion of 5-fluorouracil with respect to overall survival (OS). The other was the SPIRITS trial, which noted that $\mathrm{S}-1$ plus CDDP has been identified as the standard chemotherapy regimen for advanced or recurrent gastric cancer in Japan (8).

Docetaxel has shown promising activity in gastric cancer, both as a monotherapy (10) and in combination with other agents (11-13). Phase I and II studies of combination therapy were performed with docetaxel and S-1 for patients with advanced or recurrent gastric cancer $(14,15)$. In the phase II study, the overall response rate was $56.3 \%$ (95\% confidence interval $[\mathrm{CI}], 38-66 \%)$ and the median survival time period was 14.3 months (95\% CI, 10.27-20.3 months).

On the basis of these results, a phase III study (JACCRO GC-03 study) comparing S-1 alone with the combination therapy (docetaxel and S-1) was launched. This is a prospective, multicenter (Korea and Japan), multinational, randomized study of patients with advanced gastric cancer. In total, 638 patients were recruited, and the final results are anticipated in 2010. Previous studies reported that the docetaxel and S-1 combination has good clinical efficacy with acceptable toxicity when administered as the first-line treatment for patients with advanced gastric cancer $(16,17)$.

Systemic chemotherapy is the key treatment for patients with stage IV gastric cancer. In some cases, combination chemotherapy (CDDP plus S-1 and docetaxel plus S-1) results 
Table I. Patient characteristics of the adjuvant surgery for advanced gastric cancer.

\begin{tabular}{|c|c|c|c|c|c|c|c|c|c|c|}
\hline \multirow[t]{2}{*}{ Case no. } & \multirow[t]{2}{*}{ Age } & \multirow[t]{2}{*}{ Gender } & \multicolumn{5}{|c|}{ Clinical stage } & \multirow{2}{*}{$\begin{array}{l}\text { No. of } \\
\text { cycles }\end{array}$} & \multirow[t]{2}{*}{ Response } & \multirow{2}{*}{$\begin{array}{l}\text { Surgical } \\
\text { curability }\end{array}$} \\
\hline & & & $\mathrm{T}$ & $\mathrm{N}$ & $\mathrm{H}$ & $\mathrm{M}$ & $\mathrm{P}$ & & & \\
\hline 1 & 70 & $\mathrm{M}$ & 3 & 1 & 1 & 0 & 0 & 3 & SD & $\mathrm{C}$ \\
\hline 2 & 73 & $\mathrm{M}$ & 3 & 2 & 1 & 0 & 0 & 3 & SD & $\mathrm{C}$ \\
\hline 3 & 64 & $\mathrm{~F}$ & 3 & 2 & 1 & 0 & 0 & 3 & PR & B \\
\hline 4 & 61 & $\mathrm{~F}$ & 3 & 2 & 1 & 0 & 0 & 3 & PR & $\mathrm{B}$ \\
\hline 5 & 72 & M & 3 & 2 & 1 & 0 & 0 & 4 & PR & $\mathrm{B}$ \\
\hline 6 & 63 & M & 3 & 2 & 1 & 0 & 0 & 6 & PR & $\mathrm{B}$ \\
\hline 7 & 65 & M & 1 & 3 & 0 & 0 & 1 & 4 & PR & $\mathrm{C}$ \\
\hline 8 & 41 & M & 2 & 3 & 0 & 0 & 1 & 3 & PR & $\mathrm{B}$ \\
\hline 9 & 49 & $\mathrm{~F}$ & 3 & 1 & 0 & 0 & 1 & 6 & PR & $\mathrm{C}$ \\
\hline 10 & 45 & M & 4 & 1 & 0 & 0 & 1 & 4 & PR & $\mathrm{B}$ \\
\hline 11 & 27 & M & 4 & 3 & 0 & 0 & 1 & 3 & PR & $\mathrm{C}$ \\
\hline 12 & 65 & M & 3 & 1 & 0 & 0 & 1 & 5 & PR & $\mathrm{B}$ \\
\hline 13 & 25 & $\mathrm{~F}$ & 3 & 3 & 0 & 0 & 0 & 3 & SD & $\mathrm{C}$ \\
\hline 14 & 78 & $\mathrm{~F}$ & 3 & 3 & 0 & 0 & 0 & 5 & PR & $\mathrm{C}$ \\
\hline 15 & 75 & $\mathrm{M}$ & 4 & 3 & 0 & 0 & 0 & 2 & PR & B \\
\hline 16 & 36 & $\mathrm{M}$ & 3 & 3 & 0 & 0 & 0 & 5 & PR & B \\
\hline 17 & 70 & $\mathrm{M}$ & 3 & 3 & 0 & 1 & 0 & 5 & PR & $\mathrm{C}$ \\
\hline 18 & 61 & $\mathrm{~F}$ & 3 & 3 & 0 & 0 & 0 & 5 & PR & B \\
\hline 19 & 67 & $\mathrm{M}$ & 3 & 3 & 0 & 0 & 0 & 7 & PR & B \\
\hline 20 & 56 & $\mathrm{M}$ & 3 & 3 & 1 & 1 & 0 & 11 & PR & $\mathrm{C}$ \\
\hline
\end{tabular}

in long-term survival in clinical practice. In selected cases, additional (adjuvant) surgery may result in further long-term survival. Surgical resection was classified as curative (no evidence of remaining disease after surgery) or palliative (remaining disease after surgery). In this context, adjuvant surgery aimed to be curative, as opposed to palliative, after the response to chemotherapy. Following adjuvant surgery, chemotherapy is required.

This study therefore aimed to evaluate the efficacy of adjuvant surgery after response to the chemotherapy for advanced gastric cancer.

\section{Materials and methods}

Patients. The study included 20 advanced gastric cancer patients treated between September 2003 and December 2008 at Hiroshima University Hospital. Patients were diagnosed with unresectable advanced gastric cancer and had undergone gastrectomies following the response to chemotherapy with docetaxel and S-1.

Treatment regimen. S-1 $\left(80 \mathrm{mg} / \mathrm{m}^{2}\right)$ was administered orally after morning and evening meals for 2 weeks, followed by a drug-free interval of 1 week (one cycle). Docetaxel $\left(40 \mathrm{mg} / \mathrm{m}^{2}\right)$ was diluted in $100 \mathrm{ml}$ of $0.9 \%$ saline and administered as a 1-h infusion on the morning of day 1 of each cycle (i.e., every 3 weeks). The docetaxel infusion was started simultaneously with the $\mathrm{S}-1$ administration. Dexamethasone $(8 \mathrm{mg})$ was infused $1 \mathrm{~h}$ before the docetaxel administration. Responses were classified according to the Response Evaluation Criteria in Solid Tumors
(RECIST) guidelines (18) and the guidelines of the Japanese Gastric Cancer Association (19). To assess responses every 4-6 weeks, the tumor area was measured using a 5-mm slice computed tomography scan for all measurable lesions. Toxicity was graded according to the Common Terminology Criteria for Adverse Events (CTCAE) version 3.0 (20).

Indications for adjuvant surgery. The indications for adjuvant surgery (after the response to chemotherapy) are that curative resection (not palliative) is anticipated based on the response to chemotherapy. Thus, indications such as the absence of other distant metastases, including peritoneal dissemination, extensive lymph node metastases or lung metastasis and feasible macroscopically complete removal of liver deposits are considered. Other indications include curative resection which is anticipated despite a poor response to chemotherapy (stable disease).

Statistical analysis. OS was calculated from the date of chemotherapy initiation to the date patients succumbed to all causes or the latest follow-up. The median OS was estimated using the Kaplan-Meier method. Multivariate analysis of prognostic factors was performed by the Cox proportional method to evaluate the effects of prognostic factors on patient survival. $\mathrm{P}<0.05$ was considered to indicate a statistically significant difference.

\section{Results}

Patient characteristics. A total of 20 advanced gastric cancer patients were treated from September 2003 to December 2008 


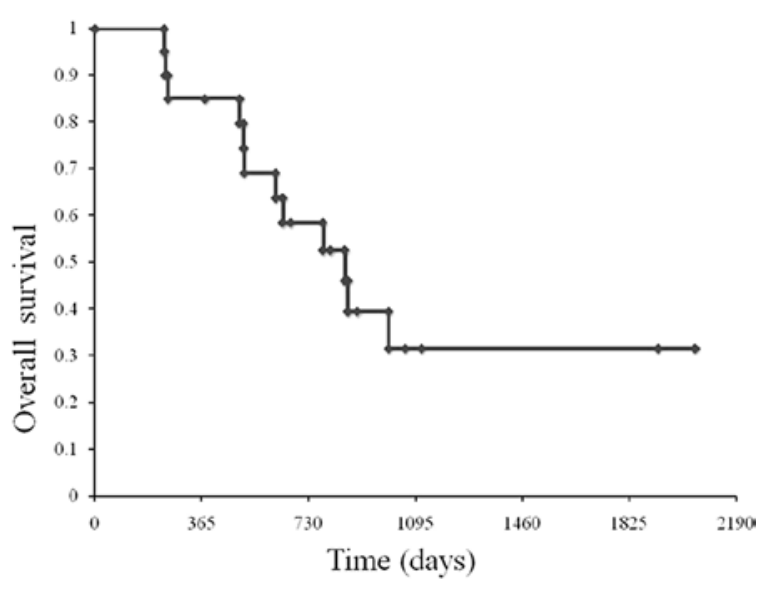

Figure 1. Overall survival. At a median follow-up of 980 days, OS was 855 days.

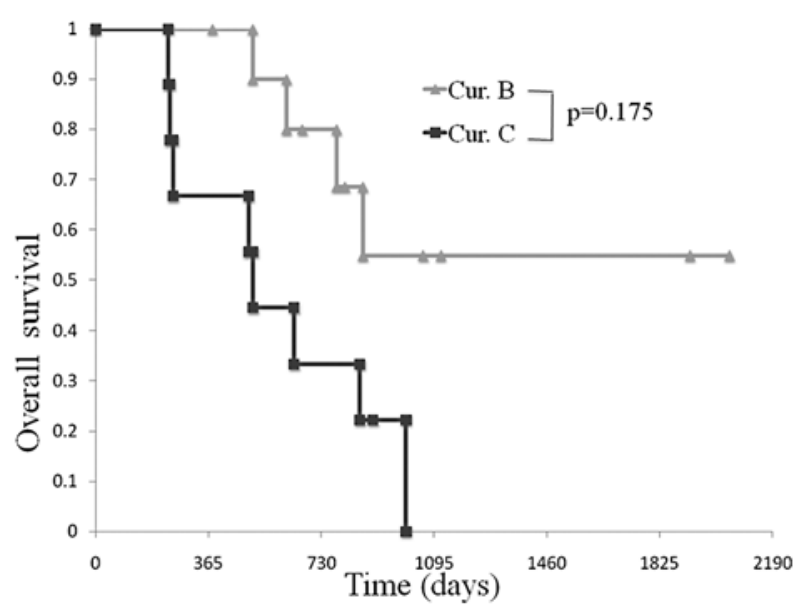

Figure 2. Comparison of OS in patients between the curative resection and palliative groups. A 2- and 3-year survival was observed in 80 and $54.9 \%$ of patients, respectively, following macroscopic curative surgery. In the palliative group, the median OS was 510 days, but a 3-year survival was not observed.

at Hiroshima University Hospital. Table I shows the patient characteristics. The median age was 58.8 years (range 25-73). All 20 patients showed a performance status of 0 or 1 . The median number of cycles administered per patient was 4.4 (range 2-11). Patients were assessable for response. No complete response was noted, while 17 patients $(85 \%)$ had partial responses (PR) and 3 (15\%) had stable disease (SD). The patients had been diagnosed as resectable following chemotherapy. However, in 9 patients, peritoneal dissemination or tumor cells in peritoneal fluid on cytological analysis were found during surgery, resulting in palliative surgery. At a median follow-up of 980 days, OS was 855 days (Fig. 1).

Comparison of OS in patients between the curative resection and palliative groups. A 2- and 3-year survival was observed in 80 and $54.9 \%$ of patients, respectively, following macroscopic curative surgery. In the palliative group, the median OS was 510 days, but a 3 -year survival was not observed (Fig. 2).

Comparison of OS in patients between the partial response and stable disease group. In the partial response group, the median

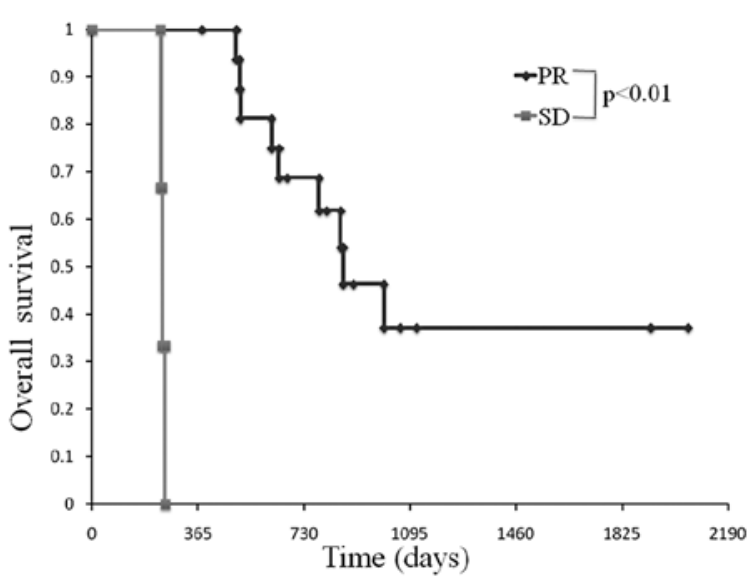

Figure 3. Comparison of OS in patients between the partial response and stable disease groups. In the partial response group, the median OS was 865 days and a 3 -year survival was observed in $37 \%$ of patients. One-year survival was not observed in the stable disease group.

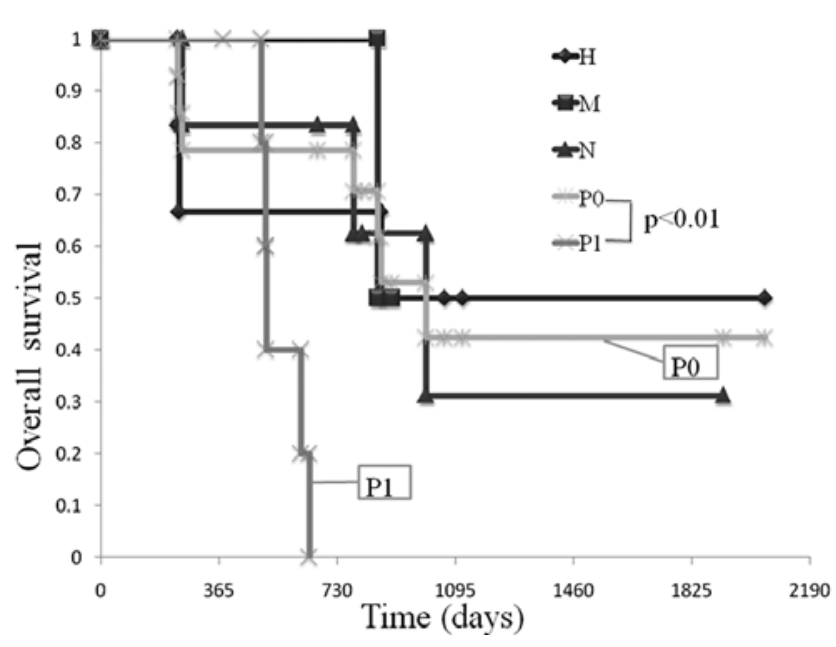

Figure 4. Comparison of OS among the various unresectable factors (liver metastasis, $\mathrm{H}$ factor; peritoneal dissemination, $\mathrm{P}$ factor; lymph node metastasis, $\mathrm{N}$ factor). The median OS in patients with the $\mathrm{H}$ factor was 865 days, while that in patients with the $\mathrm{P}$ factor was 510 days.

OS was 865 days and a 3-year survival was observed in $37 \%$ of patients. One-year survival was not observed in the stable disease group. OS in the partial response group was statistically more prolonged than the stable disease group (Fig. 3).

Comparison of OS in patients amonng the various unresectable factors (liver metastasis, $H$ factor; peritoneal dissemination, $P$ factor; lymph node metastasis, $N$ factor). A comparison was made of OS among the various unresectable factors. The median OS in patients with the $\mathrm{H}$ factor was 865 days, while that in patients with the $\mathrm{P}$ factor was 510 days (Fig. 4).

\section{Discussion}

Various treatment regimens have been developed $(5,6,9)$ and have improved the survival of patients with advanced or recurrent gastric cancer (21). The rationale for the design of the combination therapy of docetaxel and S-1 was the significant 
laboratory and clinical anti-tumor activity of both docetaxel and 5-fluorouracil in gastric cancer, their synergistic activity in vivo and the relative lack of overlapping toxicities (22). Takahashi $e t$ $a l$ reported increased anti-tumor activity in combination therapy with docetaxel and S-1 using gastric cancer xenografts (23). Wada et al reported that docetaxel and S-1 combination therapy showed synergistic effects by modulating the expression of the metabolic enzymes of 5-fluorouracil, including thymidylate synthase, dihydropyrimidine dehydrogenase and orotate phosphoribosyl transferase in human gastric cell lines (24). In a phase II study of docetaxel and S-1 combination therapy, the median time to tumor progression was 7.3 months $(95 \% \mathrm{CI}$, 4.3-10.0 months) (15).

Although progress in chemotherapy has resulted in longterm survival, a number of patients require treatment changes or a reduction in dose levels due to drug resistance or adverse events. Such patients are required to change to another regimen (second- or third-line).

When chemotherapy has produced transient tumor regression and curative surgery may be accomplished, curative resection in selected patients is occasionally associated with prolonged survival. This type of surgery is referred to as adjuvant surgery. The difference between adjuvant surgery and neoadjuvant chemotherapy is their respective indications.

Neoadjuvant (preoperative) chemotherapy is an investigational option. Its rationale is based on the difficulty of performing an R0 resection in patients with locally advanced tumors and the high risk of micrometastatic disease in these patients. Neoadjuvant treatments aim to: i) downstage the primary tumor, resulting in a higher R0 resection rate, and ii) simultaneously treat micrometastases at an early stage (25-28).

Although the basic treatment in cases of advanced gastric cancer is chemotherapy, it is insufficient. Additionally, adjuvant surgery is preferred for patients with a favorable response to chemotherapy.

The timing of such surgery may occur at the point when the tumor is reduced, but prior to the appearance of drug resistance. Empirically speaking, in gastric cancer, metastasis occurs immediately after surgery, at the time of drug resistance.

This study aimed to evaluate the efficacy of adjuvant surgery after response to the chemotherapy for advanced gastric cancer. The OS of patients in the partial response and curative resection groups was prolonged. The survival of patients with $\mathrm{H}$ or $\mathrm{N}$ factor was also prolonged, if they received curative surgery. However, the survival of patients with $\mathrm{P}$ factor was not prolonged. Adjuvant surgery is effective in gastric cancer patients diagnosed as stage IV in the case of liver or distant lymph node metastasis, but not peritoneal dissemination.

To prove the efficacy of adjuvant surgery, a randomized controlled study is necessary. Numerous obstacles remain to be addressed regarding the selection of combination drugs (S-1 plus CDDP, S-1 plus docetaxel and docetaxel plus CDDP plus 5-fluorouracil), the timing of adjuvant surgery and the selection of chemotherapy after surgery.

\section{References}

1. Kamangar F, Dores GM and Anderson WF: Patterns of cancer incidence, mortality, and prevalence across five continents: defining priorities to reduce cancer disparities in different geographic regions of the world. J Clin Oncol 24: 2137-2150, 2006.
2. Inoue $M$ and Tsugane $S$ : Epidemiology of gastric cancer in Japan. Postgrad Med J 81: 419-424, 2005.

3. Murad AM, Santiago FF, Petroianu A, Rocha PR, Rodrigues MA and Rausch M: Modified therapy with 5-fluorouracil, doxorubicin, and methotrexate in advanced gastric cancer. Cancer 72 : 37-41, 1993.

4. Glimelius B, Ekstrom K, Hoffman K, et al: Randomized comparison between chemotherapy plus best supportive care with best supportive care in advanced gastric cancer. Ann Oncol 8: 163-168, 1997.

5. MacDonald JS, Schein PS, Woolley PV, et al: 5-fluorouracil, doxorubicin, and mitomycin (fam) combination chemotherapy for advanced gastric cancer. Ann Intern Med 93: 533-536, 1980.

6. Findlay M, Cunningham D, Norman A, et al: A phase II study in advanced gastro-esophageal cancer using epirubicin and cisplatin in combination with continuous infusion 5-fluorouracil (ECF). Ann Oncol 5: 609-616, 1994.

7. Ohtsu A, Shimada Y, Shirao K, et al: Randomized phase III trial of fluorouracil alone vs. fluorouracil plus cisplatin vs. uracil and tegafur plus mitomycin in patients with unresectable, advanced gastric cancer: the Japan Clinical Oncology Group Study (JCOG9205). J Clin Oncol 21: 54-59, 2003.

8. Koizumi W, Narahara H, Hara T, et al: S-1 plus cisplatin vs. S-1 alone for first-line treatment of advanced gastric cancer (SPIRITS trial): A phase III trial. Lancet Oncol 9: 215-221, 2008.

9. Boku N, Yamamoto S, Fukuda H, et al: Fluorouracil vs. combination of irinotecan plus cisplatin vs. S-1 in metastatic gastric cancer: a randomised phase 3 study. Lancet Oncol 10: 1063-1069, 2009.

10. Einzig AI, Neuberg D, Remick SC, et al: Phase II trial of docetaxel (taxotere) in patients with adenocarcinoma of the upper gastrointestinal tract previously untreated with cytotoxic chemotherapy: the Eastern Cooperative Oncology Group (ECOG) results of protocol e1293. Med Oncol 13: 87-93, 1996.

11. Thuss-Patience PC, Kretzschmar A and Reichardt P: Docetaxel in the treatment of gastric cancer. Future Oncol 2: 603-620, 2006.

12. Fushida S, Fujimura T, Oyama K, Yagi Y, Kinoshita J and Ohta T: Feasibility and efficacy of preoperative chemotherapy with docetaxel, cisplatin and S-1 in gastric cancer patients with para-aortic lymph node metastases. Anticancer Drugs 20: 752-756, 2009.

13. Zang DY, Yang DH, Kim MJ, et al: Dose-finding study of docetaxel, oxaliplatin, and S-1 for patients with advanced gastric cancer. Cancer Chemother Pharmacol 64: 877-883, 2009.

14. Yoshida K, Hirabayashi N, Takiyama W, et al: Phase I study of combination therapy with S-1 and docetaxel (TXT) for advanced or recurrent gastric cancer. Anticancer Res 24: 1843-1851, 2004.

15. Yoshida K, Ninomiya M, Takakura N, et al: Phase II study of docetaxel and S-1 combination therapy for advanced or recurrent gastric cancer. Clin Cancer Res 12: 3402-3407, 2006.

16. Suzuki T, Yoshida K, Tanabe K, et al: Three advanced gastric cancer patients successfully treated by combination therapy of docetaxel and TS-1. Gan To Kagaku Ryoho 32: 509-513, 2005.

17. Tsutani Y, Ohara M, Suzuki T, et al: Docetaxel and S-1 as a first-line treatment in patients with advanced or recurrent gastric cancer. Anticancer Res 29: 2775-2779, 2009.

18. Eisenhauer EA, Therasse P, Bogaerts J, et al: New response evaluation criteria in solid tumours: revised RECIST guideline (version 1.1). Eur J Cancer 45: 228-247, 2009.

19. Japanese Gastric Cancer Association: Japanese classification of gastric carcinoma - 2nd english edition. Gastric Cancer 1: 10-24, 1998.

20. Trotti A, Colevas AD, Setser A, et al: CTCAE v3.0: development of a comprehensive grading system for the adverse effects of cancer treatment. Semin Radiat Oncol 13: 176-181, 2003.

21. Tanabe K, Yoshida K, Hamai Y, et al: Clinical study of TS-1 for inoperative and recurrent gastric cancer and evaluation of long survival cases. Gan To Kagaku Ryoho 32: 1145-1148, 2005.

22. Van Den Neste E, de Valeriola D, Kerger J, et al: A phase I and pharmacokinetic study of docetaxel administered in combination with continuous intravenous infusion of 5-fluorouracil in patients with advanced solid tumors. Clin Cancer Res 6: 64-71, 2000.

23. Takahashi I, Emi Y, Kakeji Y, Uchida J, Fukushima M and Maehara Y: Increased antitumor activity in combined treatment TS-1 and docetaxel. A preclinical study using gastric cancer xenografts. Oncology 68: 130-137, 2005. 
24. Wada Y, Yoshida K, Suzuki T, et al: Synergistic effects of docetaxel and S-1 by modulating the expression of metabolic enzymes of 5-fluorouracil in human gastric cancer cell lines. Int J Cancer 119: 783-791, 2006.

25. Koizumi W, Tanabe S, Saigenji K, et al: Phase I/II study of S-1 combined with cisplatin in patients with advanced gastric cancer. Br J Cancer 89: 2207-2212, 2003.

26. Nakajima $\mathrm{T}$, Ota $\mathrm{K}$, Ishihara $\mathrm{S}$, et al: Combined intensive chemotherapy and radical surgery for incurable gastric cancer. Ann Surg Oncol 4: 203-208, 1997.
27. Chua YJ and Cunningham D: The UK NCRI MAGIC trial of perioperative chemotherapy in resectable gastric cancer: implications for clinical practice. Ann Surg Oncol 14: 2687-2690, 2007.

28. Alexander HR, Grem JL, Pass HI, et al: Neoadjuvant chemotherapy for locally advanced gastric adenocarcinoma. Oncology (Williston Park) 7: 37-53, 1993. 